\title{
Pulse rate and transit time analysis to predict hypotension events after spinal anesthesia during programmed cesarean labor
}

\author{
Juan Bolea ${ }^{1,2}$, Jesús Lázaro ${ }^{1,2}$, Eduardo Gil ${ }^{1,2}$, Eva Rovira $^{3}$, José M. Remartínez ${ }^{3}$, \\ Pablo Laguna ${ }^{1,2}$, Esther Pueyo ${ }^{1,2}$, Augusto Navarro $^{3}$, Raquel Bailón ${ }^{1,2}$ \\ ${ }^{1}$ BSICoS Group, Aragón Institute of Engineering Research (I3A), IIS Aragón, University of Zaragoza, \\ Zaragoza, Spain. \\ ${ }^{2}$ Centro de Investigación Biomédica en Red Bioingeniería, Biomateriales y Nanomedicina (CIBER-BBN), \\ Madrid, Spain. \\ ${ }^{3}$ Anaesthesia Service, Hospital Miguel Servet, Zaragoza, Spain, Faculty of Medicine, University of \\ Zaragoza, Zaragoza, Spain.
}

Abbreviated Title: PR and PTT analysis to predict hypotension

Name: Juan Bolea

Mailing address: Mariano Esquillor SN, Edif. I+D+I, Lab 4.0.05, 50018, Zaragoza, Spain.

Phone number: +34 976765468

email: jbolea@unizar.es 


\section{ABSTRACT}

Prophylactic treatment has been proved to reduce hypotension incidence after spinal anesthesia during cesarean labor. However, the use of pharmacological prophylaxis could carry out undesirable side-effects on mother and fetus. Thus, the prediction of hypotension becomes an important challenge. Hypotension events are hypothesized to be related to a malfunctioning of autonomic nervous system (ANS) regulation of blood pressure. In this work, ANS responses to positional changes of 51 pregnant women programmed for a cesarean labor were explored for hypotension prediction. Lateral and supine decubitus, and sitting position were considered while electrocardiographic and pulse photoplethysmographic signals were recorded. Features based on heart rate variability (HRV), pulse rate variability (PRV) and pulse transit time (PTT) analysis were used in a logistic regression classifier. The results showed that PRV irregularity changes, assessed by approximate entropy, from supine to lateral decubitus, and standard deviation of PTT in supine decubitus were found as the combination of features that achieved the best classification results sensitivity of $76 \%$, specificity of $70 \%$ and accuracy of $72 \%$, being normotensive the positive class. Peripheral regulation and blood pressure changes, measured by PRV and PTT analysis, could help to predict hypotension events reducing prophylactic side-effects in the low-risk population.

Keywords: Heart Rate Variability; Nonlinear analysis; Pulse rate; Pulse transit time; Hypotension; Cesarean section. 


\section{INTRODUCTION}

The amount of cesarean deliveries worldwide has been reported to increase by a global average of $12.4 \%$ from 1990 to $2014^{5}$. Although the use of spinal anesthesia has reduced mortality and morbidity during the labors ${ }^{20}$, side-effects such as hypotension and bradycardia in the mother ${ }^{25}$ and hypoxia and acidosis in the fetus ${ }^{39}$ can appear after its administration. In particular, the incidence of hypotension events has been reported between 30 and $100 \%$ of cases ${ }^{29,35}$. This wide range of incidence is associated with discrepancies in the hypotension definition and measurement techniques ${ }^{23}$.

The use of prophylaxis has led to a notable reduction in the number of hypotension events ${ }^{12}$. However, prophylaxis through sympathomimetic drugs administration can lead to a non-desired reactive hypertension with dosedependent incidence up to $82 \%$ of the patients ${ }^{2}$. Prediction of hypotension events during cesarean section would help to: 1) decrease the latency time between hypotension event and its treatment; 2) increase efficiency in the anesthetic administration to better control for the hemodynamic consequences of spinal block; 3) decrease the number needed to treat (NNT), thus avoiding unneeded pharmacological exposition and therefore reducing the derived sideeffects. Prediction of hypotension after spinal anesthesia during elective cesarean delivery has been reported in the literature ${ }^{7,9,10,15,19,21,23,40,42}$. However, results have been found to be highly dependent on the number of studied subjects, the definition of hypotensive episode, and the features considered for the analysis. 
The malfunctioning or disability of autonomic nervous system (ANS) to compensate a drop in blood pressure induced by anesthesia is one of the hypothesis underlying hypotension episodes. Besides, pregnancy alters the physiological and psychological stress response. ANS regulation on the heart can be assessed by heart rate variability (HRV) analysis. Considered as a noninvasive technique, $\mathrm{HRV}$ is derived from the surface electrocardiogram (ECG) signals, whose quantification can be done taking into account the temporal, spectral and nonlinear characteristics. Sympathetic and parasympathetic regulations are related to the HRV content analyzed in low $(0.04-0.15 \mathrm{~Hz})$ and high $(0.15-0.4 \mathrm{~Hz})$ frequency bands, respectively, during relax conditions in adult healthy subjects ${ }^{1}$. Furthermore, nonlinear HRV assessment relates changes on sympathovagal balance to regularity and complexity of $\mathrm{ANS}^{8}$.

HRV analysis was found correlated to hypotension episodes the day of surgery $6,10,19,42$. Moreover, the inclusion of an ANS elicitation protocol by postural changes during the day before the surgery has been reported to reveal a relation between ANS responsiveness and the occurrence of hypotension events $^{40}$.

The pulse rate extracted from pulse photoplethysmographic (PPG) recording represents an improvement in signal recording since only one sensor is used. This sensor could be placed in the finger or in the forehead making the recording stage suitable for the clinical practice. The analysis of pulse rate variability (PRV) provides the assessment of ANS regulation of the heart and also information about the peripheral vascular regulation ${ }^{18}$. In addition, the pulse transit time (PTT) derived from the ECG and PPG signals was reported to be related to the arterial stiffness, ANS, respiration, and, blood 
pressure ${ }^{11,26,34,38}$. An increase of PTT values has been associated with a drop in arterial blood pressure (hypotension episode) $)^{41}$. Although, several studies attempted to shed light into the role of HRV and pulse oximeter derived index analysis for predicting hypotension after spinal anesthesia for cesarean section delivery, the hypotension prediction is still a challenge $\mathrm{e}^{10,19,40,42}$.

The goal of this study is to explore pre-surgery ANS responsiveness based on HRV, PRV, and PTT indices into the prediction of hypotension events induced by spinal anesthesia during cesarean delivery. The protocol to enhance ANS elicitation includes body position change stages producing hemodynamic changes a few minutes before the surgery.

\section{MATERIALS AND METHODS}

\section{Materials}

A total of 105 pregnant women programmed for an elective cesarean section selected at the University Hospital Miguel Servet of Zaragoza, Spain. After obtaining approval from the Clinical Research Ethics Committee the parturient were recruited, informed and they signed the written consent. Indications for programmed cesarean delivery were: iterative cesarean labor; breech presentation; shoulder presentation; placenta previa; previous uterotomy and other causes. Exclusion criteria were urgent surgery, uterine contractions and maternal or fetal pathology.

The subjects were randomly selected to belong to a control group (no prophylactics were administered, 51 subjects) and to prophylactic group (54 
subjects). A prospective study of this work is based on the factors related to hypotension within the control group. Thus, subjects were grouped according to hypotensive outcome. Hypotension episode was defined as a drop in systolic arterial pressure greater than $20 \%$ from baseline value (mean of three consecutive measurements taken in lateral decubitus). 33 subjects of the control group suffered hypotension after spinal injection until birth reaching a $64.7 \%$ incidence.

Demographic data were obtained at admission. Biomedical signals were recorded in the surgical area the day of surgery. Two-lead electrocardiogram (ECG) and pulse photoplesthymogram (PPG) were recorded both at $1 \mathrm{KHz}$ during a protocol designed to enhance ANS alterations by hemodynamic changes. Protocol stages were: 7 minutes of lateral decubitus assuming baseline pregnancy conditions (LD), 7 minutes of supine decubitus increasing hemodynamic stress due to aorto-cava compression (SD), 7 minutes of sitting position (SP). Whereas LD and SD were recorded for all subjects of the database, sitting position was recorded for 40 subjects. Thus, two subgroups are defined, LD-SD and LD-SD-SP.

After recording ECG and PPG signals, all patients received an infusion of Hartman solution at $15 \mathrm{ml} / \mathrm{kg} / \mathrm{h}$. Thereafter, standardized spinal anesthesia was performed: puncture site was lumbar interspace $L 2 / 3$ or $L 3 / 4$ with the patient in a sitting position. Next, 9 to $11 \mathrm{mg}$ of hyperbaric bupivacaine anesthetic $0.5 \%$ according to patient height $(<150 \mathrm{~cm}, 9 \mathrm{mg} ; 150-165 \mathrm{~cm}, 10 \mathrm{mg} ;>165 \mathrm{~cm}, 11$ $\mathrm{mg}$ ) and $10 \mu \mathrm{g}$ fentanyl was injected via $27-\mathrm{G}$ Whitacre needle. Immediately after injection, patients were positioned supine with a left lateral tilt of about $10^{\circ}$, 
to facilitate left uterine displacement. Blood pressure was measured at 2-min intervals beginning 1 min after spinal injection.

The database of study contains demographic data recorded from the beginning of the pregnancy period until the day of admission to the hospital. These baseline characteristics are shown in Table 1 according to their hypotensive outcome.

TABLE 1. Parturient characteristic data according to hypotension outcome.

\begin{tabular}{|c|c|c|c|c|}
\hline \multirow[b]{2}{*}{ Description } & \multicolumn{2}{|c|}{ Hypotension } & \multicolumn{2}{|c|}{ Normotension } \\
\hline & $\mathrm{n}$ & mean \pm SD & $\mathrm{n}$ & Mean \pm SD \\
\hline Age (yr) & 33 & $35.27 \pm 4.69$ & 18 & $33.72 \pm 4.64$ \\
\hline Height (cm) & 33 & $161.76 \pm 5.86$ & $17^{*}$ & $161.44 \pm 5.79$ \\
\hline Weight (kg) & 33 & $73.75 \pm 10.47$ & $17^{*}$ & $74.32 \pm 10.48$ \\
\hline Pre-gestational weight (kg) & 33 & $61.93 \pm 9.90$ & $15^{*}$ & $62.46 \pm 8.94$ \\
\hline Gained weight (kg) & 33 & $11.51 \pm 4.42$ & $14^{*}$ & $11.30 \pm 3.12$ \\
\hline Pregestational BMI $\left(\mathrm{kg} / \mathrm{m}^{2}\right)$ & 33 & $23.69 \pm 3.73$ & $14^{*}$ & $23.96 \pm 3.13$ \\
\hline $\mathrm{BMI}\left(\mathrm{kg} / \mathrm{m}^{2}\right)$ & 33 & $28.24 \pm 4.13$ & $17^{*}$ & $28.53 \pm 3.90$ \\
\hline Gestational age (days) & 33 & $263.2 \pm 6.7$ & 18 & $267.6 \pm 6.3$ \\
\hline
\end{tabular}

Methods

Series to study 
Heartbeat time occurrences were detected from the ECG lead with the highest signal quality (signal to noise ratio) by a Wavelet-based ECG detector ${ }^{31}$. Ectopic beats were detected and corrected ${ }^{32}$. Then consecutive occurrence times were used to generate the beat-to-beat RR time series.

Temporal location of each PPG pulse wave was detected as the mediumamplitude point in which the amplitude has reached $50 \%$ of its maximum extracted from the PPG signal ${ }^{28}$. Ectopic and undetected pulses were identified and their corresponding pulse wave time corrected ${ }^{32}$. Then consecutive pulse wave times were used to generate the pulse to pulse (PP) time series.

Pulse transit time (PTT) is the time that the pulse wave takes to travel from one arterial point (typically, aortic valve) to another one (typically, in the periphery). It was estimated as the time interval between an R peak in ECG signal and its corresponding PPG pulse wave location time. It is also called pulse arrival time since it includes the pre-ejection period (PEP).

Respiratory rate was extracted from ECG signals by applying a QRS morphology-based technique that exploits the respiration-induced variations in QRS slopes and R-wave angle ${ }^{27}$.

\section{Temporal analysis}

RR, PP and PTT time series were used to calculate the temporal indices such as mean value $\left(\mu^{X}\right)$, standard deviation $\left(\sigma^{X}\right)$, square root of the mean squared differences of successive values $\left(R M S S D^{X}\right)$, standard deviation of differences of successive values $\left(S D S D^{X}\right)$, where $X \in\{R R, P P, P T T\}$. Median value $\left(\bar{f}^{R}\right)$, and interquartile range $\left(I Q R\left[f^{R}\right]\right)$ were computed from the respiratory rate. 


\section{Spectral analysis}

The time-invariant integral pulse frequency modulation model (TI-IPFM) was applied to estimate the modulating signals from heartbeat locations which are assumed to carry information about ANS activity once compensated for the mean heart rate effect ${ }^{4}$. In the following, these modulating signals are referred to as HRV signals. Similarly as for heartbeat locations, pulse wave occurrence times were used to compute modulating signals through the TI-IPFM model labelled as PRV signals. HRV and PRV signals were interpolated at $4 \mathrm{~Hz}$. The TI-IPFM model was not applied to the PTT signal and this series was only interpolated at $4 \mathrm{~Hz}$ to be suitable for further spectral analysis.

Welch-periodogram was used to estimate spectral properties of the HRV, PRV and PTT signals. Fixed spectral band of LF $(0.04-0.15 \mathrm{~Hz})$ and a variable HF band centered in $\bar{f}^{R}$ with a fix bandwidth of $0.25 \mathrm{~Hz}$ were used to compute the power content on each band $\left(P_{L F}^{Y}\right.$ and $\left.P_{H F}^{Y}\right)$, their normalized values $\left(P_{L F n}^{Y}\right.$ and $\left.P_{H F n}^{Y}\right)$ as well as the sympathovagal balance $\left(R_{L F / H F}^{Y}\right)$, where $Y \in$ $\{H R V, P R V, P T T\}$. The variable HF band aims to better capture parasympathetic activity since respiratory rate in pregnant women can be higher and often near the classical HF upper bound of $0.4 \mathrm{~Hz}^{3}$. However, in those cases where HF band overlapped with LF band, the lower limit of HF band was set as the upper

LF band limit. No estimation based on spectral analysis was given if $\bar{f}^{R}$ was found below $0.15 \mathrm{~Hz}$ due to the uncertain physiological interpretation of these measurements.

Nonlinear analysis 
Correlation dimension, $D_{2}$, sample and approximate entropy, SampEn and $A p E n_{\max }$, were selected as nonlinear indices whose interpretation is related to complexity and irregularity ${ }^{7}$. In a previous study, nonlinear HRV indices were proposed to be HR-corrected by selecting fix time interval and later interpolating instead of fixing the number of heartbeats ${ }^{8}$. Thus, RR, PP and PTT time series were interpolated at $2 \mathrm{~Hz}$ to be suitable to compute nonlinear indices attenuating the $\mathrm{HR}$ effect.

\section{Statistical analysis}

ANS activity was assessed by analyzing the variations of HR, PR and PTT signals recorded few minutes before surgery on each protocol stage, lateral decubitus, supine decubitus, and sitting position. Differences between body position stages were also evaluated. As an example of notation, $P_{H F}^{H R V}(S D-L D)$ will be the difference of high frequency power for HRV analysis between supine and lateral decubitus.

The normality of statistical distributions was analyzed by Kolmogorov-Smirnov test. Sensitivity analysis of features comparing hypotension with no hypotension groups was tested by T-Student test on normal distributions and by Mann-Whitney $U$ test on non-normal ones.

Due to the large amount of considered features (more than 200), only those showing a statistical sensitivity on hypotension were used as candidates for classification. A univariate statistical significance was considered for $p$ values $<$ 0.05. In this case, a post hoc Bonferroni correction was not applied since the aim is to obtain a combination of features. A set of classifiers were tested such as Logistic Regression, Naive Bayes, Nearest Neighbor, Linear and Quadratic 
Discriminant Analysis, and Supported Vector Machine to search for the best classification results. Some of these classifiers provide their outcome in discrete values of 1 or 0 depending on whether the subject belongs or not to the non-hypotensive (or normotensive) group. However, other classifiers such as the logistic regression provide their outcome as a probability of belonging to hypotension group, see eq. 1 .

$$
p=\frac{1}{1+e^{-\left(\beta_{0}+\beta_{1} X_{1}+\beta_{2} X_{2}+\cdots+\beta_{N} X_{N}\right)}},
$$

where $\beta_{o}, \ldots, \beta_{N}$ are the coefficients and $X_{1}, \ldots, X_{N}$ represent the selected $\mathrm{N}$ features. Then, a probability threshold, $p_{t h}$, determines the risk of developing hypotension.

Feature selection was done by a Greedy forward algorithm by maximizing F1 score as inclusion criteria. F1 score is defined as a measurement equilibrating the positive predictive value and sensitivity, thus improving the classification of the minority class, which, in our case, is the normotensive group. Overtraining was avoided by using the leave-one-out technique combined with bootstrapping ${ }^{14}$ (Fig 1.) and by restricting the maximum number of selected features for classification to be less than the square root of the number of subjects in the smallest group. 


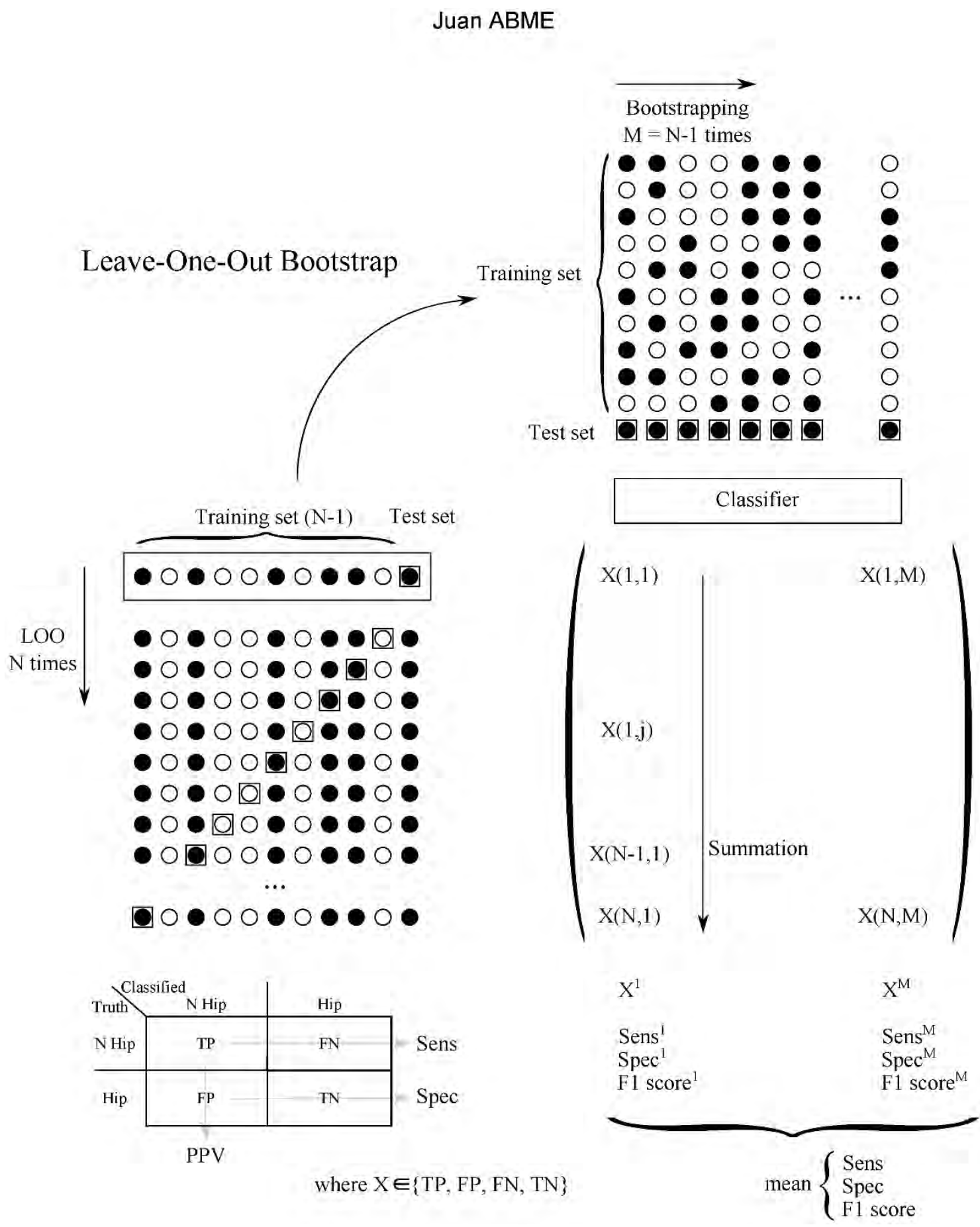

FIGURE. 1. Cross-validation scheme in which leave-one-out (LOO) technique is combined with bootstrapping to reduce the variance of the classification results. Circles represent each subject of the database whereas white and black illustrate their truly hypotension outcome as normotensive and hypotensive respectively. First, leave-one-out is applied defining a training and a test set 
(dashed rectangle). Next, bootstrapping is applied $\mathrm{N}-1$ times over this training set. Then, these bootstrapped training sets are evaluated in the classifier. Leave-one-out procedure is repeated $\mathrm{N}$ times generating the result matrix $\mathrm{X}$.

\section{RESULTS}

In Figure 2 RR, PR and PTT signals are shown for two subjects belonging to hypotensive and normotensive groups during supine position.

\section{Juan ABME}
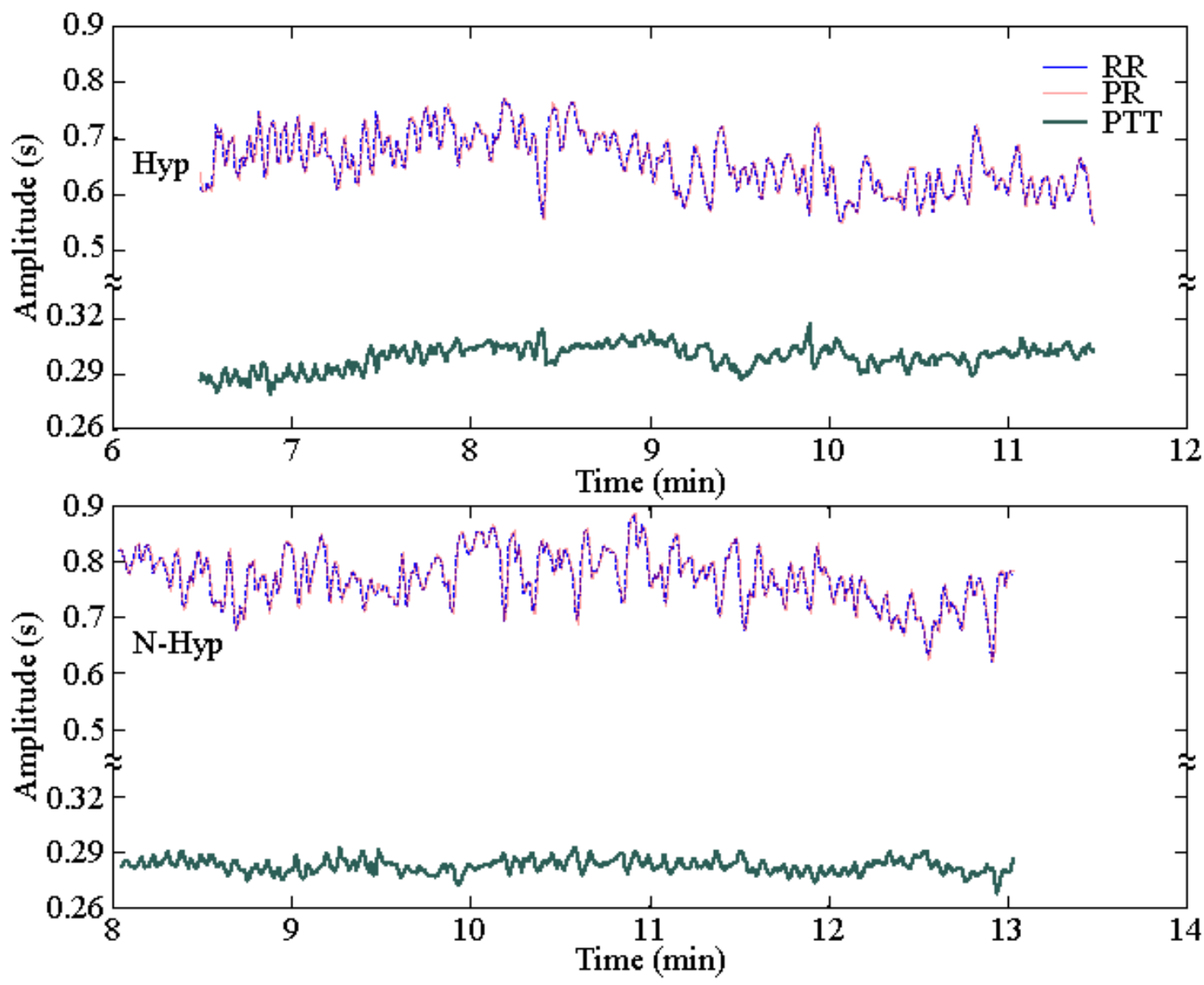
FIGURE. 2. RR, PR and PTT signals for two subjects belonging to hypotensive group (upper panel) and normotensive group (bottom panel) during supine position.

Table 2 and 3 show those indices that were found statistically significant between the two groups in LD-SD and LD-SD-SP cohorts respectively.

TABLE 2. Relation of features extracted from ECG derived respiration, RR, HRV, PR, PRV, and PTT analysis for the LD-SD cohort. Only those that were found statistically significant are shown.

\begin{tabular}{|c|c|c|c|c|c|c|c|}
\hline \multirow[b]{2}{*}{ Features } & \multicolumn{3}{|c|}{ Hyp } & \multicolumn{3}{|c|}{ N-Hyp } & \multirow[b]{2}{*}{$p$-val } \\
\hline & $\mathrm{n}$ & Mean & $\mathrm{SD}$ & $\mathrm{n}$ & Mean & SD & \\
\hline $\operatorname{SampEn} n^{R R(S D)}$ (a.u.) & 33 & 0.45 & 0.17 & 18 & 0.56 & 0.18 & 0.03 \\
\hline$S a m p E n^{R R(S D-L D)}$ (a.u.) & 33 & -0.03 & 0.16 & 18 & 0.05 & 0.11 & 0.03 \\
\hline 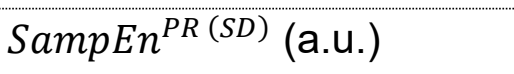 & 33 & 0.48 & 0.16 & 18 & 0.59 & 0.17 & 0.03 \\
\hline$S a m p E n^{P R(S D-L D)}$ (a.u.) & 33 & -0.04 & 0.16 & 18 & 0.06 & 0.11 & 0.02 \\
\hline$A p E n_{(\max )}^{P R(S D-L D)}$ (a.u.) & 33 & -0.04 & 0.09 & 18 & 0.03 & 0.07 & 0.01 \\
\hline$\sigma^{P T T(S D)}(\mathrm{ms})$ & 33 & 6.20 & 4.45 & 18 & 4.31 & 1.13 & 0.03 \\
\hline$P L F^{P T T(S D)}\left(\mathrm{ms}^{-2}\right)\left(\cdot 10^{-04}\right)$ & 33 & 0.15 & 0.39 & $17^{*}$ & 0.04 & 0.03 & 0.02 \\
\hline$P L F_{n}^{P T T(S D)}(\%)$ & 33 & 63.22 & 0.39 & $17^{*}$ & 51.56 & 16.3 & 0.03 \\
\hline$P L F^{P T T(S D-L D)}\left(\mathrm{ms}^{-2}\right)\left(\cdot 10^{-04}\right)$ & 33 & 0.08 & 0.24 & $17^{*}$ & 0.02 & 0.02 & 0.02 \\
\hline$R_{L F / H F}{ }^{P T T(S D)}$ (adim.) & 33 & 2.90 & 3.38 & $17^{*}$ & 1.31 & 0.84 & 0.03 \\
\hline$S a m p E n^{P T T(S D-L D)}$ (a.u.) & 33 & -0.04 & 0.21 & 18 & 0.12 & 0.21 & 0.01 \\
\hline
\end{tabular}


$\begin{array}{llllllll}I Q R\left[f^{R}\right]^{(S D)}(\mathrm{Hz}) & 33 & 0.05 & 0.04 & 18 & 0.03 & 0.03 & \mathbf{0 . 0 3}\end{array}$

(n) refers to the number of subjects on each group. ${ }^{*}$ indicates number of subjects different from 18 caused by respiratory rate overlapped to LF band. Hyp = hypotension; N-Hyp = Normotension .

$p$-values $<0.05$ were statistically significant by Mann-Whitney $U$ test.

TABLE 3. Relation of features extracted from ECG derived respiration, RR, HRV, PR, PRV, and PTT analysis for the LD-SD-SP cohort. Only those that were found statistically significant are shown.

\begin{tabular}{|c|c|c|c|c|c|c|c|}
\hline \multirow[b]{2}{*}{ Features } & \multicolumn{3}{|c|}{ Hyp } & \multicolumn{3}{|c|}{ N-Hyp } & \multirow[b]{2}{*}{$p$-val } \\
\hline & $\mathrm{n}$ & Mean & SD & $n$ & Mean & SD & \\
\hline$A p E n_{(\max )}^{R R(S P-S D)}$ (a.u.) & 28 & 0.04 & 0.12 & 12 & -0.05 & 0.11 & 0.04 \\
\hline$S a m p E n^{P R(S D-L D)}$ (a.u.) & 28 & -0.03 & 0.12 & 12 & 0.07 & 0.11 & 0.02 \\
\hline$A p E n_{(\max )} P R(S D-L D)$ (a.u.) & 28 & -0.02 & 0.07 & 12 & 0.05 & 0.06 & 0.008 \\
\hline$S a m p E n^{P R(S P-S D)}$ (a.u.) & 28 & 0.07 & 0.15 & 12 & -0.04 & 0.18 & 0.04 \\
\hline$A p E n_{(\max )} P R(S P-S D)$ (a.u.) & 28 & 0.012 & 0.11 & 12 & -0.07 & 0.13 & 0.05 \\
\hline$\sigma^{P T T(S D)}(\mathrm{ms})$ & 28 & 6.36 & 4.76 & 12 & 4.16 & 0.84 & 0.019 \\
\hline$\sigma^{P T T(S P-S D)}(\mathrm{ms})$ & 28 & -0.75 & 3.6 & 12 & 0.94 & 1.22 & 0.02 \\
\hline$P L F^{P T T(S D)}\left(\mathrm{ms}^{-2}\right)\left(\cdot 10^{-04}\right)$ & 28 & 0.17 & 0.43 & 12 & 0.03 & 0.02 & 0.01 \\
\hline$P L F_{n}^{P T T(S D)}(\%)$ & 28 & 62.4 & 16.9 & 12 & 45.9 & 15.0 & 0.009 \\
\hline$R_{L F / H F}{ }^{P T T(S D)}$ (adim.) & 28 & 2.81 & 3.41 & 12 & 0.99 & 0.59 & 0.009 \\
\hline$R_{L F / H F}{ }^{P T T}(S P-S D)$ (adim.) & $27^{*}$ & -1.56 & 2.48 & 12 & -0.14 & 0.78 & 0.02 \\
\hline $\bar{f}^{R(S P)}(\mathrm{Hz})$ & 28 & 0.29 & 0.06 & 12 & 0.26 & 0.04 & 0.04 \\
\hline
\end{tabular}


(n) refers to the number of subjects on each group. * indicates number of subjects different from 28 caused by respiratory rate overlapped to LF band. Hyp = hypotension; N-Hyp = Normotension .

$p$-values $<0.05$ were statistically significant by Mann-Whitney $U$ test.

TABLE 4. Classification results for the LD-SD cohort for the different considered classifiers. In all cases $A p E n_{(\max )}^{P R(S D-L D)}$ and $\sigma^{P T T(S D)}$ were selected by Greedy algorithm as best feature combination.

\begin{tabular}{|c|c|c|c|c|c|c|}
\hline Classifiers & \# & Sens.(\%) & Spec.(\%) & $\mathrm{PPV}(\%)$ & Acc. $(\%)$ & $\mathrm{F} 1$ \\
\hline Logistic Regression & 2 & 76.55 & 69.81 & 57.84 & 72.20 & 0.66 \\
\hline Naive Bayes & 2 & 73.78 & 58.91 & 49.75 & 64.16 & 0.59 \\
\hline Nearest Neighbor Method & 2 & 44.78 & 73.33 & 47.96 & 63.25 & 0.46 \\
\hline LDA & 2 & 41.56 & 79.64 & 49.45 & 66.20 & 0.46 \\
\hline QDA & 2 & 68.00 & 59.70 & 48.27 & 62.63 & 0.56 \\
\hline SVM (linear kernel) & 2 & 81.56 & 61.03 & 53.94 & 68.27 & 0.65 \\
\hline SVM (quadratic kernel) & 2 & 71.33 & 58.30 & 48.70 & 62.90 & 0.57 \\
\hline SVM (polynomial order 3 kernel) & 2 & 64.56 & 57.03 & 45.59 & 59.79 & 0.53 \\
\hline SVM (Radial Basis kernel) & 2 & 75.67 & 60.48 & 51.52 & 65.84 & 0.61 \\
\hline SVM (Multilayer perceptron kernel) & 2 & 77.67 & 59.76 & 50.92 & 66.08 & 0.62 \\
\hline
\end{tabular}

(\#) number of features, (Sens.) sensitivity, (Spec.) specificity, (PPV) positive predictive value, (Acc.) accuracy, and, F1 score. 
Table 4 shows the classification results for the different tested classifiers. For all classifiers $A p E n_{(\max )}{ }^{P R(S D-L D)}$ and $\sigma^{P T T(S D)}$ were selected as the combination of features that achieved the best classification results in the LDSD cohort. The classifier showing the highest F1 score was the logistic regression.

Subsets of features from the two studied cohorts (LD-SD and LD-SD-SP) and a third cohort $\left(\right.$ LD-SD $\left.{ }^{1}\right)$ that matches the same subjects from LD-SD-SP group discarding the SP position were evaluated for classification using the logistic regression classifier. The classification results are shown in Table 5. It is worth noting that the features $A p E n_{(\max )}^{P R(S D-L D)}$ and $\sigma^{P T T(S D)}$ were selected by the classifier in the three cohorts and $\bar{f}^{R(S P)}$ was added in the set for the LD-SD-SP cohort. The parameters of the logistic models for the different studied cohorts can be found in Table 6 .

TABLE 5. Classification results considering ECG derived respiration, RR, HRV, PR, PRV, and PTT analysis features for the three subgroups of the database depending on protocol segments.

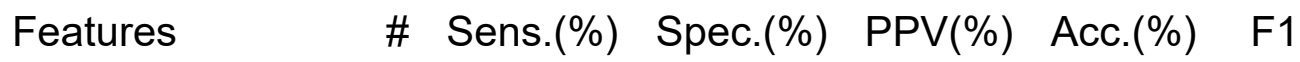

LD-SD (33 vs 18)

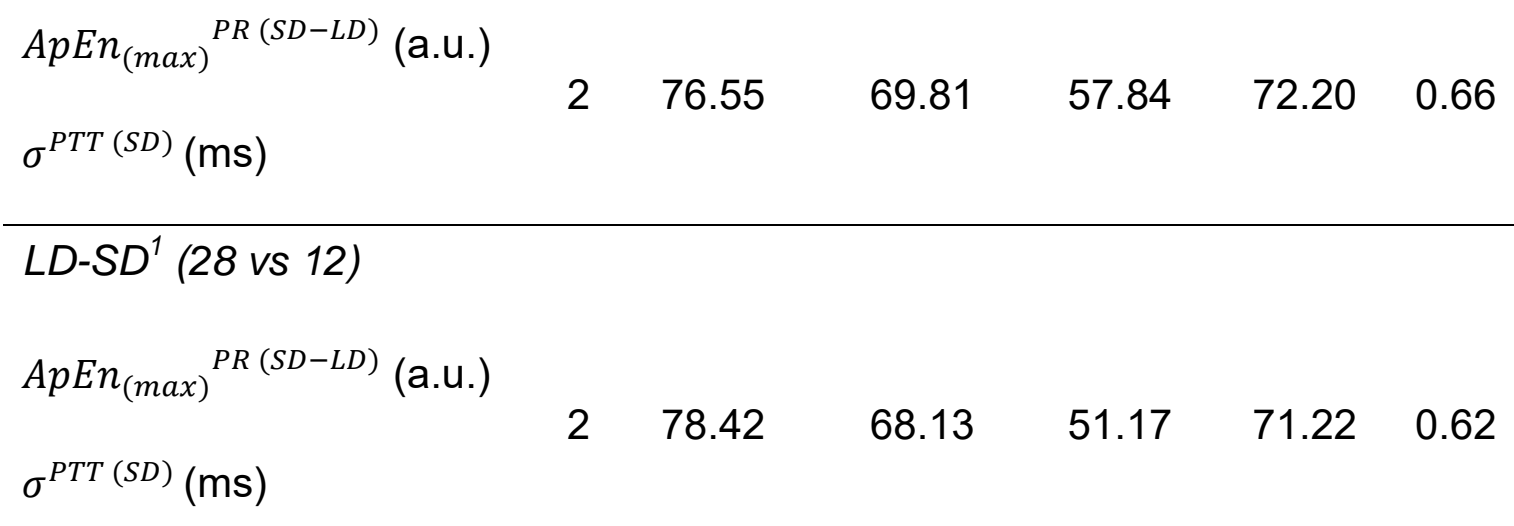


$L D-S D-S P(28$ vs 12)

$A p E n_{(\max )}^{P R(S D-L D)}$ (a.u.)

$\begin{array}{lllllll}\sigma^{P T T}(S D)(\mathrm{ms}) & 3 & 75.44 & 74.85 & 56.66 & 75.03 & 0.65\end{array}$

$\bar{f}^{R(S P)}(\mathrm{Hz})$

(\#) number of features, (Sens.) sensitivity, (Spec.) specificity, (PPV) positive predictive value, (Acc.) accuracy, and, F1 score.

\section{Juan ABME}

a) $\mathrm{LD}-\mathrm{SD}$
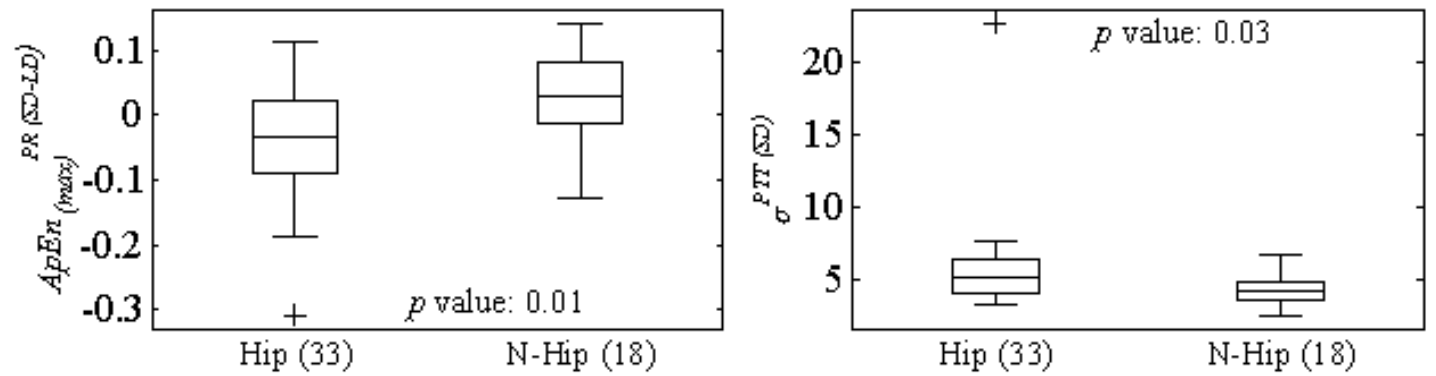

b) LD-SD-SP
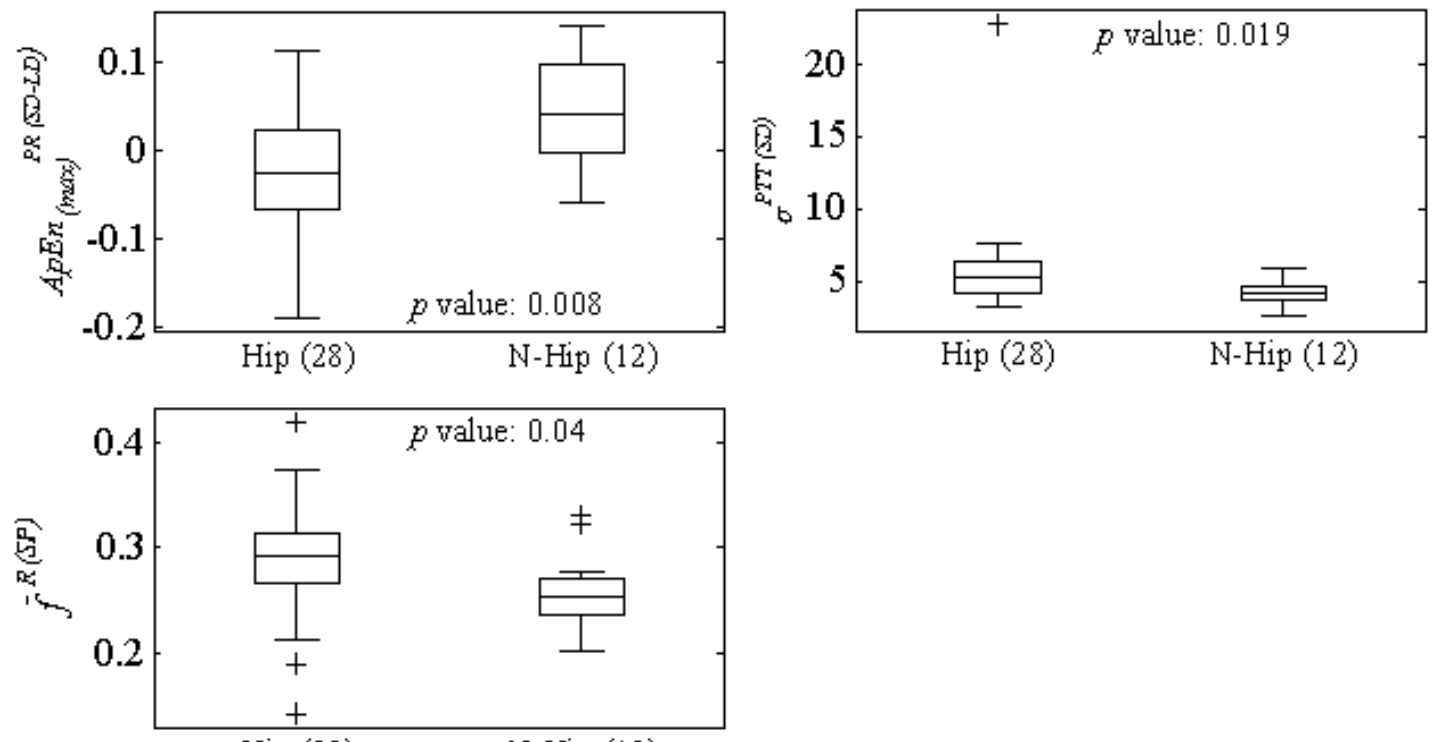

Hip (28)

N-Hip (12) 
FIGURE. 3. Boxplots of the features finally selected by classification procedure for a) LD-SD and b) LD-SD-SP cohorts.

TABLE 6. Logistic regression parameters for the different cohorts.

\begin{tabular}{lccc}
\hline \multicolumn{1}{c}{ Logistic reg. parameters } & LD-SD & LD-SD $^{1}$ & LD-SD-SP \\
& $(33$ vs 18) & (28 vs 12) & (28 vs 12) \\
\hline$\hat{\beta}_{o}$ & 0.306 & 0.446 & 3.13 \\
$\hat{\beta}_{1}\left[\sigma^{P T T(S D)}\right]$ & -0.38 & -0.479 & -0.555 \\
$\hat{\beta}_{2}\left[A p E n_{(\max )}^{P R(S D-L D)}\right]$ & 8.36 & 10.48 & -3.753 \\
$\hat{\beta}_{3}\left[\bar{f}^{R(S P)}\right]$ & - & - & -10.55 \\
$p_{t h}$ & 0.342 & 0.268 & 0.344 \\
\hline
\end{tabular}

Where $\hat{\beta}^{\prime} s$ are the coefficients and in [] is represented the corresponding feature following eq. 1. $p_{t h}$ is the probability threshold of risk of hypotension.

\section{DISCUSSION}

Hypotension episodes after spinal anesthesia during cesarean delivery were hypothesized to be more frequent in patients with high level of stress. Proximity of surgery jointed to the last period of pregnancy could make the ANS response an estimate of the risk of hypotension ${ }^{13,36}$. To evaluate this hypothesis, the database used in this study was recorded following a specific protocol enhancing the ANS response due to hemodynamic changes. Beyond the use of an ANS elicitation protocol (body position changes), which has been proved 
to be helpful to identify women at risk of severe hypotension events ${ }^{24}$, one of the novelties of this study lies on the inclusion of the PTT signal analysis as an alternative to blood pressure measurement that can be more easily obtained in clinical practice ${ }^{34}$. Furthermore, PTT signal provides beat-to-beat information of arterial stiffness, respiration whereas this information is missed in BP measurements by cuff recordings. Improving the accuracy in predicting hypotension events to avoid the side-effects that pharmacological prophylaxis may produce in the mother and fetus in the low risk patient is the goal of our study. However, the performance obtained by predicting hypotension could be affected by hypotension events detection since after spinal injection BP was measured every 2 minutes. To minimize this effect, clinical hypotension was also considered when women showed symptoms of dizziness, nausea or discomfort.

In this work, the interaction of respiration on the ANS modulation of the heart is also taken into account. This effect, known as RSA, is reflected in the HRV, PRV and PTT through the HF band corresponding to the parasympathetic activity $^{21}$. Variability analysis, in particular HRV analysis, could lead to misleading interpretation depending on respiratory rate ${ }^{3}$. As mentioned above, stress induced by surgery proximity may produce an increase in the respiratory rates (boundaries of HF band) and also compression of diaphragm. In such case, parasympathetic activity is reflected in HRV at higher frequencies and thus classical HF band is not capable of capturing the information properly. Therefore, we propose to guide variability analysis by respiration defining an HF band centered in the respiration rate. 
The analysis of HRV and PRV were found to be highly correlated to each other in agreement with the results of Gil et $\mathrm{al}^{17}$ (correlation results are not shown in the manuscript). The evaluation on lateral decubitus, considered as a basal condition, revealed no differences between both groups of study when analyzing RR, HRV or PR, PRV. However, it was found a decreased tendency on the standard deviation of PTT and on its complexity for the normotensive group with respect to the one who suffered hypotension.

The hemodynamic stress was generated by supine decubitus position via aortocava compression. The analyses of RR, HRV and PR, PRV showed similar temporal and spectral values for both groups without statistical significance. Nevertheless, the normalized low frequency power of PTT was found increased and therefore, greater sympathetic activity ${ }^{16}$ was recorded in the hypotensive group with respect to the normotensive group. On the other hand, greater and significant changes were observed for SampEn pointing out to a decrease in the time series irregularity of RR and PR for hypotensive group. PTT analysis revealed an increase in the amplitude variation values in the hypotensive group, see Fig. 2. On the one hand, PTT measure is affected by pulse wave velocity, which is related to BP. On the other hand, in the present study PTT values include the PEP, pre-ejection period, which was reported to experiment changes under enhanced sympathetic conditions ${ }^{22,30}$. PEP was also reported to represent from $12 \%$ to $35 \%$ of the PTT ${ }^{38}$ and its variations could have a strong influence on the variations of the PTT measure ${ }^{33}$. Variations on PEP could help in the prediction of hypotensive episodes since those variations are enhanced in the same way as the ones in pulse wave velocity ones increasing the PTT discrimination power. Although one limitation of this study is the 
impossibility of separating those effects, the studied PTT measure can be easily obtained in the clinical routine and has discriminant power between hypotensive and normotensive groups.

The responsiveness of subjects through body position changes was explored by differences between supine and lateral decubitus analysis values. The statistical differences between hypotensive and normotensive group were more notable in terms of irregularity for RR, PR and also for PTT evaluating the position change. Thus, this position change modifies the venous return and affects the ANS activity as well as arterial pressure and stiffness. The differences of approximate entropy values between supine and lateral decubitus computed on PR achieved the lowest p-value when comparing both groups of study. On the contrary, same index computed on RR signal did not reveal statistical differences, so hemodynamic changes affect more peripheral regulation (blood pressure) than heart regulation (heart rate). The difference of standard deviation of PTT values observed between groups may be measuring the capacity of ANS to react in the case of a drop in blood pressure. The normotensive group could present higher reaction range (lower $\sigma^{P T T(S D)}$ values) whereas the hypotensive group shows a narrow margin (higher $\sigma^{P T T}(S D)$ values) to reach the hypothetical physiological maximum value at which triggers a hypotension event.

The studied features that presented statistical differences comparing normotensive and hypotensive groups were selected as candidates for hypotension classification. Because of the high incidence ratio of hypotension events $(64.7 \%$ of cases), it is mandatory to predict the normotensive cases; otherwise looking for hypotensive cases we can achieve higher accuracy values 
but neglecting the other class. Thus, classification performance was carried out by evaluating the positive predictive value and sensitivity related to the normotensive group. Several classifiers were tested and the one providing the best classification results was selected, see Table 4 . In all cases the Greedy algorithm selected $A p E n_{(\max )}^{P R(S D-L D)}$ and $\sigma^{P T T(S D)}$ as the best combination of features. Regarding the F1 score measurement, the logistic regression classifier provided the best classification results for the LD-SD cohort: $76.55 \%$ of sensitivity, $69.81 \%$ of specificity, $57.84 \%$ of positive predictive value, and $72.2 \%$ of accuracy. Then, hereinafter classification results are referred to the logistic regression classifier.

Sitting position was also included in the protocol to enhance the ANS activity when the database recording was in process. The first 20 subjects ( 11 of the control group) were not asked to be in the sitting position while recording the signals. All the analysis procedure was duplicated considering this position on a subset of the whole database (LD-SD-SP), see Table 5. In this cohort, $\bar{f}^{R}(S P)$ was selected increasing the classification performance with respect to the LD$\mathrm{SD}^{1}$ cohort. On the other hand, the comparison between sitting position and lateral decubitus did not reveal any index capable of distinguishing both groups of study. The variation in the sympathetic activity caused by sitting position with respect to the other body positions was apparently not enough to generate notable changes on the studied indices.

In the study of Brenck et al. risk factors for developing hypotension based on population characteristics were studied over 500 pregnant women, achieving accuracy around $60 \%{ }^{9}$. We performed a test combining the demographic data 
with the features derived from the ECG and PPG signals and the classification results using logistic regression were superior to only considering ECG and PPG-derived indices. In particular, the Greedy algorithm selected BMI and the standard deviation of PTT values during supine position, altogether leading to classification performance characterized by $82.5 \%$ sensitivity, $67.9 \%$ specificity, and $72.9 \%$ of accuracy. Based on this, it is reasonable to suggest that the combination of demographic data and features derived from ECG and PPG signals can lead to better classification results. However, this statement should be validated over an extended database. Several studies attempted to shed light into the role of HRV and pulse oximeter derived index analysis for predicting hypotension after spinal anesthesia for cesarean section delivery ${ }^{10,19,40,42}$. Despite of Yokose et al. found heart rate as the only predictor obtaining an area under the ROC curve (AUC) of $0.68^{42}$, this feature did not show predictive power in the database analyzed in our study. In other study carried out by Sakata and coworkers, the importance of including postural changes into the recording protocol to enhance hemodynamic changes and ANS response was highlighted achieving an area under the ROC curve of $0.76^{40}$. There, sympathovagal ratio, LF/HF, was reported to be increased in those parturient who developed hypotension. In our case, similar trend was found for the LF/HF ratio through the PTT signal (Hyp: 2.9 \pm 3.38 versus No-Hyp: $1.31 \pm 0.84$ in LD-SD cohort and Hyp: $2.81 \pm 3.41$ versus No-Hyp: $0.99 \pm 0.59$ in LD-SD-SP cohort), see Table 2 and 3. Ghabach et al. reported a relationship between approximate entropy and antenatal weight gain ${ }^{15}$. Assuming that this position was lateral decubitus, which represents the basal position without any extra hemodynamic stress, the results we found are in disagreement with the 
ones reported by Ghabach. In our study no statistical differences were found in the weight gain (results shown in Table 1) nor in approximate entropy during lateral position (Hyp: $0.87 \pm 0.08$ ); $\mathrm{N}$-Hyp: $0.85 \pm 0.1$ and $p$-value: 0.95 , values corresponding to LD-SD cohort). Although in the work of Yokose et al. neither HRV nor PRV were useful to prospectively relate the analysis results to hypotension events ${ }^{42}$, our study provides contrary insights. Lack of standardization on hypotension definition and the low number of subjects become a limitation leading into controversial results. Thus, future studies on a larger database should be explored.

Regarding the classification results obtained in this study, first, we would like to focus our attention into sensitivity values. Normotensive cases were defined as positive cases for classification since this group is minority and also the one who may suffer from anesthetic side-effects in case detection fails. Among normotensives (18 women), the $76.55 \%$ of them $(\sim 14)$ were correctly classified, thus only the $25 \%(\sim 4)$ could present these anesthetic side-effects. On the contrary, among hypotensive cases (33 women), the $69.8 \%$ ( 23) were correctly classified whereas the $31.2 \%(\sim 10)$ were classified as normotensives and they will suffer from hypotension caused by anesthesia but they will be detected and treated for when the event appears. The benefits of our classification results were highlighted being only 4 out of 55 women could present undesirable, but avoidable by proper prediction, side-effect in mother and fetus. The classifier presented in this manuscript could help to better assist clinicians to take decisions. It is of course considered that the higher accuracy the better, with typically over than $80 \%$ as a plausible clinical target, so it still 
has limitations to base clinical decisions only on classification results as the ones presented in this study.

PR and PTT analysis could help to predict hypotension events after spinal anesthesia during cesarean section extending the description of ANS regulation and its response to postural changes. These analyses may indicate the role of peripheral regulation and blood pressure changes in order to avoid the undesirable side-effects of prophylactic administration in the low-risk population.

\section{ACKNOWLEDGMENTS}

This work was funded under projects TEC2013-42140-R, TIN2013-41998-R and TIN2014-53567-R by MINECO (Spain) and by BSICOS Group (T96) from Government of Aragón and European Social Fund (EU), and by ISCIII, Spain, through Project PI10/ 02851(FIS). CIBER is a center of the Instituto de Salud Carlos III in assistance from the European Regional Development Fund. The computation was performed by the ICTS "NANBIOSIS", more specifically by the High Performance Computing Unit of the CIBER in Bioengineering, Biomaterials \& Nanomedicine (CIBER-BBN) at the University of Zaragoza.

\section{REFERENCES}


1. Akselrod, S., D. Gordon, F. A. Ubel, D. C. Shannon, A. C. Berger, and R. J. Cohen. Power spectrum analysis of heart rate fluctuation: a quantitative probe of beat-to-beat cardiovascular control. Science 213(4504):220-2, 1981.

2. Allen, T. K., R. B. George, W. D. White, H. A. Muir, and A. S. Habib. A double-blind, placebo-controlled trial of four fixed rate infusion regimens of phenylephrine for hemodynamic support during spinal anesthesia for cesarean delivery. Anesth. Analg. 111(5):1221-9, 2010.

3. Bailon, R., P. Laguna, L. Mainardi, and L. Sornmo. Analysis of heart rate variability using time-varying frequency bands based on respiratory frequency. Conf.Proc. IEEE Eng. Med. Biol. Soc. 2007; 6675-8, 2007.

4. Bailón, R., G. Laouini, C. Grao, M. Orini, P. Laguna, and O. Meste. The integral pulse frequency modulation model with time-varying threshold: application to heart rate variability analysis during exercise stress testing. IEEE Trans. Biomed. Eng. 58(3):642-52, 2011.

5. Betrán, A. P., J. Ye, A.-B. Moller, J. Zhang, A. M. Gülmezoglu, and M. R. Torloni. The Increasing Trend in Caesarean Section Rates: Global, Regional and National Estimates: 1990-2014. PLoS One 11(2):e0148343, 2016.

6. Bolea, J., R. Bailón, E. Rovira, J. M. Remartínez, P. Laguna, and A. Navarro. Heart rate variability in pregnant women before programmed Cesarean intervention. Conf. Proc. IFMBE 2014. 41:710-713, 2014.

7. Bolea, J., P. Laguna, J. M. Remartínez, E. Rovira, A. Navarro, and R. Bailón. Methodological framework for estimating the correlation dimension in HRV signals. Comput. Math. Methods Med.Vol 2014, Article ID 
129248,1-11 doi:10.1155/2014/129248.

8. Bolea, J., E. Pueyo, M. Orini, and R. Bailón. Influence of heart rate in nonlinear HRV indices as a sampling rate effect evaluated on supine and standing. Front. Physiol. 7:501, 2016.

9. Brenck, F., B. Hartmann, C. Katzer, R. Obaid, D. Brüggmann, M. Benson, R. Röhrig, and A. Junger. Hypotension after spinal anesthesia for cesarean section: identification of risk factors using an anesthesia information management system. J. Clin. Monit. Comput. 23(2):85-92, 2009.

10. Chamchad, D., V. A. Arkoosh, J. C. Horrow, J. L. Buxbaum, I. Izrailtyan, L. Nakhamchik, D. Hoyer, and J. Y. Kresh. Using Heart Rate Variability to Stratify Risk of Obstetric Patients Undergoing Spinal Anesthesia. Anesth. Analg. 99(6):1818-1821, 2004.

11. Chua, C. P., and C. Heneghan. Pulse transit time-derived respiratory parameters and their variability across sleep stages. Proc. Annu. Int. Conf.Proc. IEEE Eng. Med. Biol. Soc. 2005; 6:6153-6, 2005.

12. Cleary-Goldman, J., M. Negron, J. Scott, R. A. Downing, W. Camann, L. Simpson, and P. Flood. Prophylactic ephedrine and combined spinal epidural: maternal blood pressure and fetal heart rate patterns. Obstet. Gynecol. 106(3):466-72, 2005.

13. Dishman, R. K., Y. Nakamura, M. E. Garcia, R. W. Thompson, A. L. Dunn, and S. N. Blair. Heart rate variability, trait anxiety, and perceived stress among physically fit men and women. Int. J. Psychophysiol. 37(2):121-33, 2000.

14. Efron, B., and R. J. Tibshirani. An introduction to the bootstrap. CRC 
press, 1994.

15. Ghabach, M. B., M. F. El-Khatib, T. G. Zreik, M. S. Matta, J. J. Mouawad, C. J. Karam, and C. M. Ayoub. Effect of weight gain during pregnancy on heart rate variability and hypotension during caesarean section under spinal anaesthesia. Anaesthesia 66(12):1106-1111, 2012.

16. Gil, E., R. Bailón, J. M. Vergara, and P. Laguna. PTT variability for discrimination of sleep apnea related decreases in the amplitude fluctuations of PPG signal in children. IEEE Trans. Biomed. Eng. 57(5):1079-88, 2010.

17. Gil, E., M. Orini, R. Bailón, J. M. Vergara, and P. Laguna. Comparative analysis between PPG variability and HRV during non-stationary tilt table test. Conf. Proc. ESGCO,1-4, 2010.

18. Gil, E., M. Orini, R. Bailón, J. M. Vergara, L. Mainardi, and P. Laguna. Photoplethysmography pulse rate variability as a surrogate measurement of heart rate variability during non-stationary conditions. Physiol. Meas. 31(9):1271-1290, 2010.

19. Hanss, R., B. Bein, T. Ledowski, M. Lehmkuhl, H. Ohnesorge, W. Scherkl, M. Steinfath, J. Scholz, and P. H. Tonner. Heart rate variability predicts severe hypotension after spinal anaesthesia for elective caeserean delivery. Anesthesiology 102(6):1086-1093, 2005.

20. Hawkins, J. L., J. Chang, S. K. Palmer, C. P. Gibbs, and W. M. Callaghan. Anesthesia-related maternal mortality in the United States: 1979-2002. Obstet. Gynecol. 117(1):69-74, 2011.

21. Hirsch, J. A., and B. Bishop. Respiratory sinus arrhythmia in humans: how breathing pattern modulates heart rate. Am. J. Physiol. 241(4):H620-9, 
1981.

22. Houtveen, J. H., P. F. C. Groot, and E. J. C. Geus. Effects of variation in posture and respiration on RSA and pre-ejection period. Psychophysiology 42(6):713-719, 2005.

23. Ilies, C., H. Kiskalt, D. Siedenhans, P. Meybohm, M. Steinfath, B. Bein, and R. Hanss. Detection of hypotension during Caesarean section with continuous non-invasive arterial pressure device or intermittent oscillometric arterial pressure measurement. Br. J. Anaesth. 109(3):413419, 2012.

24. Kinsella, S. M., and M. C. Norris. Advance prediction of hypotension at cesarean delivery under spinal anesthesia. Int. J. Obstet. Anesth. 5(1):37, 1996.

25. Kyokong, O., S. Charuluxananan, P. Sriprajittichai, T. Poomseetong, and P. Naksin. The incidence and risk factors of hypotension and bradycardia associated with spinal anesthesia. J. Med. Assoc. Thai. 89 Suppl 3:S5864, 2006

26. Laurent, S., J. Cockcroft, L. Van Bortel, P. Boutouyrie, C. Giannattasio, D. Hayoz, B. Pannier, C. Vlachopoulos, I. Wilkinson, H. Struijker-Boudier, and European Network for Non-invasive Investigation of Large Arteries. Expert consensus document on arterial stiffness: methodological issues and clinical applications. Eur. Heart J. 27(21):2588-605, 2006.

27. Lázaro, J., A. Alcaine, D. Romero, E. Gil, P. Laguna, E. Pueyo, and R. Bailón. Electrocardiogram derived respiratory rate from QRS slopes and R-wave angle. Ann. Biomed. Eng. 42(10):2072-83, 2014.

28. Lázaro, J., E. Gil, M. Vergara, and P. Laguna. Pulse Rate Variability 
Analysis for Discrimination of Sleep-Apnea-Related Decreases in the Amplitude Fluctuations of Pulse Photoplethysmographic Signal in Children. IEEE J. Biomed. Health. Inform. 18(1):240-6, 2014.

29. Maayan-Metzger, A., I. Schushan-Eisen, L. Todris, A. Etchin, and J. Kuint. Maternal hypotension during elective cesarean section and shortterm neonatal outcome. Am. J. Obstet. Gynecol. 202(1):56.e1-5, 2010.

30. Martin, S. L.-O., A. M. Carek, C.-S. Kim, H. Ashouri, O. T. Inan, J.-O. Hahn, and R. Mukkamala. Weighing Scale-Based Pulse Transit Time is a Superior Marker of Blood Pressure than Conventional Pulse Arrival Time. Sci. Rep. 6:39273, 2016.

31. Martínez, J. P., R. Almeida, S. Olmos, A. P. Rocha, and P. Laguna. A Wavelet-Based ECG Delineator Evaluation on Standard Databases. IEEE Trans. Biomed. Eng. 51(4):570-581, 2004.

32. Mateo, J., and P. Laguna. Analysis of Heart Rate Variability in the Presence of Ectopic Beats Using the Heart Timing Signal. IEEE Trans. Biomed. Eng. 50(3):334-343, 2003.

33. Muehlsteff, J., X. L. Aubert, and M. Schuett. Cuffless Estimation of Systolic Blood Pressure for Short Effort Bicycle Tests: The Prominent Role of the Pre-Ejection Period. Conf. Proc. IEEE Eng. Med. Biol. Soc. 2006; 1:5088-92, 2006.

34. Mukkamala, R., J.-O. Hahn, O. T. Inan, L. K. Mestha, C.-S. Kim, H. Töreyin, and S. Kyal. Toward Ubiquitous Blood Pressure Monitoring via Pulse Transit Time: Theory and Practice. IEEE Trans. Biomed. Eng. 62(8):1879-901, 2015.

35. Ngan Kee, W. D. Prevention of maternal hypotension after regional 
anaesthesia for caesarean section. Curr. Opin. Anaesthesiol. 23(3):3049, 2010.

36. Orbach-Zinger, S., Y. Ginosar, J. Elliston, C. Fadon, M. Abu-Lil, A. Raz, Y. Goshen-Gottstein, and L. A. Eidelman. Influence of preoperative anxiety on hypotension after spinal anaesthesia in women undergoing Caesarean delivery. Br. J. Anaesth. 109(6):943-9, 2012.

37. Payne, R. A., C. N. Symeonides, D. J. Webb, and S. R. J. Maxwell. Pulse transit time measured from the ECG: an unreliable marker of beat-to-beat blood pressure. J. Appl. Physiol. 100(1):136-141, 2006.

38. Peter, L., N. Noury, and M. Cerny. A review of methods for non-invasive and continuous blood pressure monitoring: Pulse transit time method is promising? IRBM 35:271-282, 2014.

39. Roberts, S. W., K. J. Leveno, J. E. Sidawi, M. J. Lucas, and M. A. Kelly. Fetal acidemia associated with regional anesthesia for elective cesarean delivery. Obstet. Gynecol. 85(1):79-83, 1995.

40. Sakata, K., N. Yoshimura, K. Tanabe, K. Kito, K. Nagase, and H. lida. Prediction of hypotension during spinal anesthesia for elective cesarean section by altered heart rate variability induced by postural change. Int. J. Obstet. Anesth. 29:34-38, 2017.

41. Sharwood-Smith, G., J. Bruce, and G. Drummond. Assessment of pulse transit time to indicate cardiovascular changes during obstetric spinal anaesthesia. Br. J. Anaesth. 96(1):100-5, 2006.

42. Yokose, M., T. Mihara, Y. Sugawara, and T. Goto. The predictive ability of non-invasive haemodynamic parameters for hypotension during caesarean section: a prospective observational study. Anaesthesia 
70(5):555-62, 2015. 